CAHIERS DE

NARRATOLOGIE

\section{Cahiers de Narratologie}

Analyse et théorie narratives

$36 \mid 2019$

Rhétorique et représentations de la culture mafieuse. Images, rituels, mythes et symboles

\title{
Pour une poétique de la mise en intrigue
}

Une lecture de l'ouvrage de Raphaël Baroni, Les Rouages de l'intrigue. Les outils de la narratologie postclassique pour l'analyse des textes littéraires, Genève, Slatkine, coll. « Érudition », 2017.

Ilias Yocaris

\section{OpenEdition}

Journals

Electronic version

URL: http://journals.openedition.org/narratologie/10158

ISSN: $1765-307 X$

Publisher

LIRCES

Electronic reference

Ilias Yocaris, «Pour une poétique de la mise en intrigue », Cahiers de Narratologie [Online], 36 | 2019,

Online since 20 December 2019, connection on 21 December 2019. URL : http://

journals.openedition.org/narratologie/10158

This text was automatically generated on 21 December 2019.

Article L.111-1 du Code de la propriété intellectuelle. 


\section{Pour une poétique de la mise en intrigue}

Une lecture de l'ouvrage de Raphaël Baroni, Les Rouages de l'intrigue. Les outils de la narratologie postclassique pour l'analyse des textes littéraires, Genève, Slatkine, coll. « Érudition », 2017.

\section{Ilias Yocaris}

1 Cet ouvrage (désormais RI) se situe dans la lignée d'une longue série d'études qui s'attachent toutes à analyser "[l]es dispositifs textuels qui servent à nouer ou à dénouer une intrigue » (RI, 15 ; cf. à titre indicatif Ricœur 1983, 1984, Phelan 1989, Brooks 1992, Sternberg 1993, Baroni 2007, Abbott 2007, Kukkonen 2014). La grande nouveauté du travail de Raphaël Baroni, c'est qu'il entreprend de décrire ces dispositifs en adoptant « une démarche intégrative » (RI, 182) : en effet, il met à profit pour mener à bien son projet des acquis théoriques issus de la narratologie classique (axée sur la structure des récits fictifs), la narratologie postclassique (axée sur l'articulation des structures narratives avec le contexte historique, les significations visées, la dynamique de la narration, le rôle du récepteur etc.), la linguistique textuelle et énonciative, la psycholinguistique, la poétique des mondes possibles, la didactique de la littérature etc. Malgré certaines imperfections de détail liées à des partis pris théoriques qui seront questionnés plus loin, cette démonstration constitue une avancée importante dans le domaine de la théorie littéraire, et ce pour deux raisons. D'une part elle permet d'esquisser " une véritable stylistique de la dynamique narrative » (RI, 82), dont les contours restent toutefois à préciser. D'autre part, elle fournit des schémas d'analyse assez souples pour analyser le processus de mise en intrigue "en contexte ", dans le cadre de genres narratifs et d'agencements énonciatifs très variés.

Raphaël Baroni a choisi de diviser son livre en trois parties. Dans la première, intitulée «Définir l'intrigue et ses fonctions » (RI, 23-62), il se fixe un double objectif. Dans le chapitre 1 (RI, 25-45), il tente de cerner plus nettement les contours du concept d'« intrigue ", qui sont assez flous. Comme il le montre, ce concept a pu être utilisé pour désigner : 
3 (1) Une séquence événementielle (désignée également, au gré des terminologies utilisées, par les termes fabula ou histoire), autrement dit «à la trame de l'histoire dans ses dimensions chronologique et causale » (RI, 31).

4 (2) Une séquence textuelle (également désignée par les termes sujet, récit ou discours), en d'autres termes «à la manière dont les informations concernant l'histoire sont effectivement présentées dans le récit » (ibid.).

5 (3) Un dispositif textuel « dont la fonction est d'inscrire les événements racontés dans une totalité intelligible et de leur conférer un sens » (ibid.).

6 (4) Un dispositif textuel « dont la fonction est d'intriguer le lecteur » (ibid.).

7 Baroni propose de restreindre le sens du vocable "intrigue», en l'utilisant exclusivement pour désigner l'acception (4) et en le dissociant notamment de (3) (acception pour laquelle il propose d'utiliser le terme configuration). Ainsi définie, l'intrigue apparaît à la base " comme une matrice de possibilités dont la fonction est de susciter un désir cognitif » (RI, 39). Les stratégies discursives visant à multiplier ces possibilités pour intriguer le lecteur correspondent à ce qu'il est convenu d'appeler le «nœud de l'intrigue ». Cette définition (RI, 40-41) peut à bon droit sembler trop vague, mais sera précisée plus loin dans le texte : elle permet d'envisager les récits à intrigue comme des «suite[s] orientée[s] de virtualités qui s'articulent les unes aux autres, à la manière d'un arbre horizontal » (RI, 42).

Le chapitre 2 (RI, 47-62) se propose de «[r]éhabiliter la lecture pour l'intrigue » (RI, 47). On tente d'y montrer que l'étude des procédés discursifs mis en œuvre pour intriguer le lecteur a des prolongements didactiques très importants, dans la mesure où elle permet de revaloriser la « lecture naïve » des textes littéraires qui est celle du grand public et des élèves du secondaire peu attirés par la littérature en général. En effet, les dispositifs textuels utilisés pour intriguer le lecteur contribuent par définition à instaurer une relation personnelle au texte reposant sur un large éventail d'affects de toutes sortes (l'émotion, le plaisir, l'admiration, la curiosité, le suspense...). Pour tout dire, ils sont à l'origine d'une "expérience immersive » (RI, 52) puisqu'ils permettent à un lecteur donné, fût-il peu compétent sur le plan technique, de reconstituer mentalement le monde possible esquissé dans le récit qu'il a sous les yeux et de s'y plonger en se mettant à la place des personnages. Une telle démarche est fort avantageuse d'un point de vue éducatif, d'autant que son apport est multiple : elle remplit à la fois une fonction cognitive, une fonction adaptative et même une fonction éthique, puisqu'elle accroît par définition « notre capacité à nous intéresser au sort des autres » (RI, 59), le fameux care cher aux philosophes anglo-saxons.

9 La deuxième partie de l'ouvrage (pp. 63-137) est intitulée « les rouages de l'intrigue », et concentre l'essentiel de la démonstration proposée : on y étudie successivement les modalités de déploiement de l'intrigue, les paramètres proprement narratologiques liés à ces modalités et les processus langagiers permettant de moduler la tension narrative. Le chap. 3 (pp. 65-80) aborde dans un premier temps la structuration séquentielle des textes mis en intrigue, en montrant que ceux-ci comportent de façon prototypique deux phases : le nouement, qui génère « une tension susceptible d'emporter le lecteur dans sa progression textuelle comme un fleuve » (RI, 69), et le dénouement, qui permet d'évacuer cette tension. R. Baroni insiste sur le fait que le dénouement «est optionnel et ne constitue que l'une des virtualités de l'histoire» (RI, 67), un récit pouvant fort bien se terminer après le nouement de l'intrigue. Il entreprend ensuite l'étude des 
dispositifs textuels permettant de générer de la tension narrative en impliquant le lecteur dans le déroulement du récit: comme il l'explique, ces dispositifs jouent essentiellement sur la curiosité et le suspense.

10 Le chap. 4 (pp. 81-115), fort consistant, propose une reconfiguration des approches narratologiques héritées de la tradition structuraliste, afin de « décrire les structures narratives en les reliant aux fonctions qu'elles sont susceptibles de remplir dans l'interaction entre le texte et le lecteur » (RI, 81). L'objectif affiché est de montrer qu'il existe des affinités entre certains types de formes narratives et certains types de fonctions, afin de faire ressortir l'inépuisable variété des processus langagiers mis en œuvre pour générer de la tension narrative. Dans cette perspective, on étudie successivement les fonctions dévolues à la caractérisation des personnages, la situation temporelle du narrateur par rapport à l'histoire qu'il raconte, la construction textuelle du point de vue, l'agencement temporel des événements par le discours et la segmentation des récits fictifs en épisodes ou en chapitres.

11 Dans le chap. 5 (pp. 117-137), R. Baroni s'attache à étudier dans le détail le support langagier de la mise en intrigue : comme il l'explique, il existe à la base (mis à part le cas des récits au futur simple) trois modes narratifs différenciés, articulés respectivement autour du passé simple, du passé composé et du présent. Après avoir détaillé les implications stylistiques liées à l'utilisation de ces «temps pivot[s]» (RI, 120), il s'attarde sur les différentes fonctions de l'imparfait, les « récits virtuels» (RI, 130) enchâssés dans la trame narrative qui esquissent autant de possibles fictionnels non réalisés et l'incarnation (embodiment) du lecteur dans le monde raconté, fruit d'un « décentrement déictique » (RI,132) typique des récits mimétiques en général.

Moins dense et moins homogène, la troisième et dernière partie des Rouages de l'intrigue (pp. 139-179), intitulée "L'intrigue dans le texte», permet de tester le dispositif théorique élaboré dans les pages précédentes, en l'utilisant pour analyser trois récits fictifs : Derborence de Charles-Ferdinand Ramuz (1934), Les Gommes d'Alain Robbe-Grillet (1953) et Le Roi Cophetua de Julien Gracq (1970). L'étude de Derborence (chap. 6, pp. 141-156) permet de mettre en évidence « la fonction esthétique remplie par la mise en intrigue » (RI, 155), mais aussi l'intrication entre le parcours biographique de Ramuz, la réception de son œuvre et les techniques narratives qu'il met en place pour intriguer le lecteur. Les développements consacrés au Roi Cophetua (chap. 7, pp. 157-166) montrent en substance que l'intrigue gracquienne est avant tout la manifestation stylisée "d'un regard posé sur le monde » (RI, 158), comme en témoignent notamment le choix des caractérisants, la restriction du volume des informations livrées sur la "servantemaitresse " et les invariants isotopiques observables dans les passages où elle est évoquée (attente, impatience, incertitude etc.) : l'effet cumulatif de tous ces procédés est une érotisation de l'intrigue, tension narrative et désir sexuel apparaissant en somme comme les deux faces d'une même pièce. Enfin, l'analyse des Gommes (chap. 8, pp. 167-179) met au jour les procédés liés à la déconstruction des stéréotypes du roman policier et du roman d'espionnage, dans le cadre d'une esthétique de la dissonance qui repose sur « un mélange subtil de répétition et d'innovation » (RI, 178).

13 Voici donc dans ses grandes lignes la démonstration entreprise par R. Baroni. Quel est le bilan que l'on peut en dresser quand on l'examine de façon critique ? L'apport global de son ouvrage est plus que conséquent : en effet, il réussit à n'en point douter le tour de force de renouveler la narratologie classique en repensant les schémas théoriques qui la sous-tendent et en l'articulant pour la première fois avec la pragma-stylistique 
contemporaine. Or, cette avancée méthodologique est d'une importance décisive parce qu'elle permet de créer des outils d'analyse technique très efficaces, dont certains peuvent être utilisés pour analyser non seulement les fictions littéraires mais aussi les fictions filmiques. Par ailleurs, l'étude des procédés utilisés pour générer de la tension narrative ouvre des perspectives théoriques nouvelles, dans la mesure où elle projette un éclairage bienvenu sur un objet d'étude délaissé à ce jour (cf. Yocaris $2016: 65,372$ ) par la stylistique littéraire : l'impact proprement esthétique des œuvres d'art verbales.

Il n'y a pas lieu de cacher toutefois que Les Rouages de l'intrigue suscitent aussi un certain nombre de réserves, qui, sans remettre fondamentalement en question la validité de l'ouvrage, laissent tout de même le lecteur assez perplexe par moments. En quoi consistent ces réserves? Force est de constater que l'approche intégrative des récits fictifs mise en œuvre par R. Baroni reste malgré tout incomplète, puisque, comme il le reconnaît lui-même, il est «loin d'avoir épuisé tous les outils que la linguistique, l'analyse du discours, la narratologie, la sémiologie ou la sociologie ont à offrir à l'analyse textuelle de la tension narrative » (RI, 137). Baroni souligne ainsi qu'il aurait pu aborder dans cette optique des processus langagiers comme l'utilisation d'adverbes temporels, la structuration isotopique des textes mis en intrigue, le recours à une syntaxe expressive ou l'usage de pronoms personnels, ce à quoi on peut ajouter, en vrac, le choix et la disposition des figures de style, l'emploi du discours implicite, la mise en place de dispositifs narratifs polyphoniques etc. Or, cette incomplétude n'est pas due uniquement à une volonté d'alléger l'ouvrage en s'en tenant à l'essentiel de la démonstration proposée : elle a également partie liée avec l'angle d'approche théorique adopté par R. Baroni, qui limite indûment (à notre sens) la portée de sa méthode d'analyse textuelle et génère des distorsions théoriques assez regrettables.

D'où vient la difficulté en l'occurrence? Le couplage de la narratologie et de la didactique mis en œuvre dans Les Rouages de l'intrigue permet certes de mener une réflexion approfondie portant à la fois sur l'analyse et l'enseignement du fait littéraire, acquis considérable s'il en est. Il y a cependant un prix à payer pour cet acquis, et il nous paraît à vrai dire assez élevé : l'ouvrage se voit ainsi plombé par un cortège assez invraisemblable d'approximations, de contre-vérités et d'affirmations discutables à l'emporte-pièce qui renvoient aux pires poncifs du pédagogisme scolaire ${ }^{1}$. Il n'y a pas lieu de lister ici ces poncifs un par un, on retiendra simplement qu'ils sont tous articulés autour de la fameuse maxime stipulant que «l'élève doit être au centre du système éducatif ». En effet, et c'est tout sauf un hasard, l'approche de la mise en intrigue défendue par Baroni est intégralement axée sur la réception des récits fictifs, en faisant fi de leur (contexte de) production et en circonscrivant de surcroît la réception dans le milieu scolaire ${ }^{2}$, domaine de prédilection des didacticiens toutes tendances confondues. Or, une méthode d'analyse ainsi paramétrée comporte nécessairement des aspects réducteurs...

16 Tout d'abord, elle empêche forcément de mettre en place une stylistique de la tension narrative pleinement intégrative, puisque cette opération requiert par définition des compétences techniques et des repérages langagiers inaccessibles à des nonspécialistes - et a fortiori des apprenants. Ensuite, elle minore par définition l'importance des paramètres liés à la stratégie discursive auctoriale dont la mise en intrigue est le fruit : le repérage de ces paramètres nécessitant une lecture " experte " censée détourner les élèves du discours littéraire ${ }^{3}$, on choisit tout bonnement de les ignorer! Ainsi, force est de constater par exemple que l'étude de la mise en intrigue 
dans Les Gommes reste très superficielle: exclusivement focalisé sur la réception du texte et son impact émotionnel immédiat, R. Baroni se borne à lister les procédés visant en somme à susciter l'intérêt du lecteur et à lui procurer du plaisir. Or, le déploiement du récit robbe-grilletien est constitutivement déterminé par des paramètres tout autres, liés (pour l'essentiel) au projet littéraire néo-romanesque qui le sous-tend. Ainsi, la déconstruction des stéréotypes liés au roman policier et les références parodiques au mythe d'CEdipe s'inscrivent dans un projet conceptuel plus vaste visant à affaiblir la "coupure dualiste" entre observant et observé qui constituait un des fondements du roman réaliste du XIXe siècle (honni par les Nouveaux Romanciers), puisqu'elle légitimait la tentative de "décrire le monde comme il est»: dans Les Gommes comme dans Æedipe roi, la ligne de démarcation entre «observant» (l'enquêteur menant des investigations sur un crime) et "observé » (le criminel qu'il tente de débusquer) disparait, puisque l'enquêteur commet lui-même le crime qu'il doit élucider... Pour tout dire, donc, un tel agencement narratif vise in fine à déconstruire "l'image d'un univers stable, cohérent, continu, univoque, entièrement déchiffrable " (Robbe-Grillet 1963 : 31), autrement dit la vision du monde « vernienne » qui nous a été léguée par le rationalisme positiviste du XIXe siècle. Les choix narratifs liés à la mise en intrigue dans Les Gommes visent donc avant tout à suggérer au lecteur qu'une telle vision du monde est devenue périmée et que le discours romanesque doit nécessairement en tenir compte : si, pour quelque raison que ce soit, on ne prend pas ce paramètre en considération, il devient tout simplement impossible de décrire de façon adéquate le fonctionnement du texte robbe-grilletien.

On pourrait certes affirmer que de telles objections portent au fond sur la manière dont R. Baroni envisage l'étude des textes littéraires, et non pas la mise en intrigue considérée en soi comme objet d'étude. Est-ce vraiment le cas? Nous n'en sommes pas persuadé. En effet, il y a tout lieu de soupçonner que le parti pris pédagogique adopté par Baroni engendre aussi des distorsions théoriques portant sur la manière dont il définit le concept même d'intrigue: on s'en souvient, l'intrigue est à ses yeux un dispositif textuel visant à intriguer le lecteur, définition axée (encore et toujours) sur la réception des récits fictifs. Or, ce choix méthodologique peut à bon droit sembler contestable, parce qu'il permet d'évacuer subrepticement une conception plus globalisante et plus synthétique de l'intrigue, celle qui se trouve - comme par hasard - au cœur de la production des fictions littéraires et filmiques: qu'est-ce à dire? Comme on l'a vu, Baroni écarte l'idée que l'intrigue stricto sensu soit un « dynamisme intégrateur qui tire une histoire une et complète d'un divers d'incidents, autant dire transforme ce divers en une histoire une et complète " (Ricœur 1984:18; italiques de Ricœur) et évoque à ce sujet un simple processus de " configuration » : de la sorte, il a tout loisir d'étudier les procédés liés à la mise en intrigue en les dissociant artificiellement de la stratégie discursive dont ils sont par définition le fruit...

À ces considérations méthodologiques on peut également ajouter certaines réserves purement techniques, portant sur les rapports entre régulation de la tension narrative et construction textuelle du point de vue : dans Les Rouages de l'intrigue, la question est envisagée en utilisant comme base de travail la théorie genettienne des focalisations, choix qui est certes naturel de la part d'un narratologue mais n'en soulève pas moins des problèmes insolubles. Prenant judicieusement acte des critiques formulées par A. Rabatel $(1997,1998)$ vis-à-vis du modèle genettien, R. Baroni propose en définitive une reconfiguration complète de ce modèle, axée sur la trichotomie [récit focalisé] vs [récit à focalisation restreinte] vs [récit à focalisation élargie ou non focalisé] (cf. RI, 101 et 
passim). Il se trouve toutefois qu'une telle trichotomie n'est pas vraiment satisfaisante, puisqu'elle apparait comme un compromis bancal entre l'approche genettienne (que l'on se refuse manifestement à abandonner tout à fait) et l'approche rabatélienne (que l'on ne parvient pas dès lors à exploiter pleinement). En effet, comme l'explique Baroni, la «focalisation restreinte" n'est rien d'autre qu'un avatar de la «focalisation externe" genettienne, la "focalisation élargie» venant se substituer, elle, à la «focalisation zéro ». Or, il est utile de rappeler qu'Alain Rabatel remet radicalement en question les concepts même de "focalisation zéro » et de "focalisation externe ", sur la base de critères strictement linguistiques dont il semble impossible de faire l'économie - du moins jusqu'à la preuve du contraire ${ }^{4}$. Dès lors, il appert que le dispositif théorique élaboré par R. Baroni devra sûrement faire l'objet d'une reconfiguration appropriée sur ce point précis. Ainsi, il faudra sans doute évacuer à terme les catégorisations genettiennes, tout en intégrant (cf. Rabatel 1998) les concepts rabatéliens de "profondeur", de "volume» et de «vision»: ces derniers sont effectivement liés à des paramètres discursifs de toutes sortes qui jouent un rôle crucial dans la modulation de la tension narrative.

\section{BIBLIOGRAPHY}

\section{Références bibliographiques}

Porter H. Abbott (2007) : «Story, Plot, and Narration », in Herman David dir., The Cambridge

Companion to Narrative, Cambridge, Cambridge University Press, pp. 39-51.

Raphaël Baroni (2007) : La Tension narrative. Suspense, curiosité et surprise, Paris, Seuil.

Peter Brooks (1992 [1984]) : Reading for the Plot. Design and Intention in Narrative, Cambridge \&

Londres, Harvard University Press.

Jérôme David (2012) : « Le premier degré de la littérature », LHT, 9, http://www.fabula.org/lht/9/ david.html.

Gérard Genette (1972) : Figures III, Paris, Seuil, coll. « Poétique ».

- (1983) : Nouveau discours du récit, Paris, Seuil, coll. « Poétique ».

Karin Kukkonen (2014) : « Plot », in Peter Hühn et al. dirs, Handbook of Narratology, Berlin \& New York, de Gruyter, pp. 1-21.

James Phelan (1989) : Reading People, Reading Plots : Character, Progression, and the Interpretation of Narrative, Chicago, University of Chicago Press.

Alain Rabatel (1997) : Une Histoire du point de vue, Metz, CELTED.

- (1998) : La Construction textuelle du point de vue, Lausanne, Delachaux \& Niestlé.

Paul Ricœur (1983) : Temps et récit, tome I, L'Intrigue et le récit historique, Paris, Seuil.

- (1984) Temps et récit, tome II, La Configuration dans le récit de fiction, Paris, Seuil.

Alain Robbe-Grillet (1963) : Pour un Nouveau Roman, Paris, Minuit, coll. « Critique ». 
Meir Sternberg (1993 [1978]) : Expositional Modes and Temporal Ordering in Fiction, Bloomington, Indiana University Press.

Ilias Yocaris (2016) : Style et semiosis littéraire, Paris, Classiques Garnier, coll. « Investigations stylistiques ».

\section{NOTES}

1. L'analyste un tant soit peu familiarisé avec la praxis du commentaire stylistique universitaire et les réalités de l'enseignement scolaire découvre non sans amusement un certain nombre de stéréotypes qui confinent à ce qu'on pourrait appeler le folklore pédagogique. Ainsi par exemple, à en croire certains didacticiens derrière lesquels R. Baroni s'abrite avec une prudence tout à fait louable, la «lecture disciplinée et dépassionnée» (RI, 49) censée être privilégiée par "l'institution scolaire » (ibid.), autrement dit une lecture critique des textes littéraires envisagés comme des sommes d'artefacts formels, n'aurait d'autre effet que de détourner les élèves du discours littéraire : «Il ne s'agit pas simplement d'apprendre à lire un roman, mais il s'agit de lire autre chose et autrement: cesser de lire des livres faciles de manière frivole pour analyser sérieusement des œuvres difficiles. On nous apprend à découvrir les classiques et à les décortiquer, à les soumettre à la question, à sortir de l'illusion du monde raconté pour prendre en considération la matérialité de l'écriture et pour admirer le style inimitable des grands écrivains. On apprend, pour gagner de la distinction, à désirer des romans austères, forcément dénués de suspense ou de rebondissements, à glorifier la soi-disant négation de l'intrigue, ce rejet que l'on croit reconnaître à chaque coin de page dans les chefs-d'œuvre du siècle passé » (ibid.; italiques du texte). Mieux encore, cette lecture "savante » ou « experte » des récits fictifs s'opposerait à la lecture " ordinaire », en fonction d'une série de couples oppositionnels du plus bel effet : «le dévoilement contre la duperie ; la saisie contre la passivité ; la pénétration virile contre l'accueil féminin » (David 2012; cité in RI, 48-49). Enfin, dernière pièce du puzzle, la lecture ordinaire aurait été "imposée à l'expérience esthétique par l'Église, l'École ou la bienséance, lorsque les femmes, les enfants ou les pauvres se sont mis à lire » (David 2012; italiques du texte ; cité in RI, 49). Bien entendu, de telles affirmations ne résistent pas une seule seconde à l'analyse... Tout d'abord, l'expérience du terrain montre hélas que l'enseignement secondaire en France se trouve actuellement à des années-lumière de toute approche formaliste des œuvres littéraires: on privilégie au contraire un thématisme des plus réducteurs qui ne prend nullement en considération des paramètres comme "la matérialité de l'écriture » ou le «style inimitable des grands écrivains ». Ainsi, l'enseignement du fait littéraire se limite de plus en plus dans les faits à l'étude paraphrastique d'œuvres intégrales ou de groupements de textes isolés de leur contexte littéraire et historique et abordés sous un angle essentiellement lexical, l'énonciation, les figures de style, la métrique (dans les textes versifiés) et la syntaxe étant souvent réduites à la portion congrue : dès lors, la perspective s'inverse, et il appert qu'il faut surtout réhabiliter les approches formalistes, qui risquent sinon de devenir une espèce en voie de disparition... Quant aux "romans austères, forcément dénués de suspense ou de rebondissements » censés être l'ordinaire des élèves du secondaire, ceux-ci tendent à être peu à peu évacués des programmes, sans autre forme de procès : quand on interroge par exemple les étudiants titulaires d'un baccalauréat littéraire qui ont opté pour la filière Lettres à l'université, on constate régulièrement que la plupart d'entre eux n'ont jamais travaillé à l'école sur des œuvres comme Du côté de chez Swann, La Jalousie ou même L'Éducation sentimentale, voire ignorent à peu près tout de leur contenu! Pour ce qui est maintenant des élucubrations sur l'opposition entre "lecture experte» et "lecture ordinaire", celles-ci sont, au mieux, dépourvues de fondement... Ainsi, on ne voit pas en vertu de quoi (sinon d'une équivalence fantasmée entre 
pénétration intellectuelle et pénétration érotique) la lecture «experte » serait par définition viriliste et "genrée », d'autant qu'elle est tout aussi bien pratiquée dans le domaine de la narratologie par des femmes dont les travaux ont fait date (comme Mieke Bal ou Dorrit Cohn) : poussera-t-on le ridicule jusqu'à affirmer que celles-ci auraient intériorisé un modus operandi typiquement masculin ? Et comment expliquer dès lors le fait que le recours non critique à la «lecture ordinaire » suscite toujours de très fortes réserves méthodologiques au sein du milieu universitaire, en dépit de la féminisation massive des effectifs dans les facultés de Lettres qui aurait dû engendrer de nouvelles normes comportementales et méthodologiques? Par ailleurs, on passe commodément sous silence la fonction émancipatrice de la "lecture experte ", qui permet justement, quand ses principes ont été bien assimilés, de remettre en question la bienséance ou encore l'autorité de l'Église et de l'École, ce qui n'est nullement le cas de la « lecture ordinaire »! Enfin, faire de la lecture ordinaire l'apanage des femmes, des enfants et des pauvres est une pure vue de l'esprit, infondée sur le plan scientifique et témoignant qui plus est d'un mépris insondable à l'égard des « classes dominées ». De telles affirmations auraient dû être étayées par des études montrant qu'il existe une corrélation directe entre le sexe ou le niveau des revenus et le type de lecture («experte» ou "ordinaire») privilégié par un individu donné : quelles sont les études en question? On aimerait bien le savoir. De plus, l'expérience du terrain montre que la lecture "experte » est accessible à tous les élèves, pour peu qu'on dispose des moyens requis (et des compétences adéquates) pour les former correctement et que le contexte s'y prête : c'est parce que ces conditions sont rarement réunies désormais dans la pratique que la lecture «experte» a périclité (n'en déplaise à certains pédagogues) dans l'enseignement secondaire, et non pas parce qu'elle serait intrinsèquement élitiste...

2. C'est tout sauf un hasard si l'on évoque les récits fictifs lus « en classe » $(R I, 83) \ldots$

3. Cf. supra, n. 1 .

4. Rabatel pose plus précisément deux questions vouées (sans doute) à rester sans réponse :

(a) Qui perçoit en définitive le référent fictionnel dans le cadre d'une séquence narrative en focalisation zéro? Genette affirme que dans les séquences de ce genre il y a en réalité "partage entre focalisation variable [= juxtaposition de focalisations internes axées sur plusieurs personnages différents] et non focalisation [= focalisation zéro stricto sensu, absence de tout foyer de perspective] 》(Genette 1972 : 208). D'où l'équivalence proposée dans Nouveau discours du récit: «focalisation zéro = focalisation variable, et parfois zéro » (Genette 1983: 49). Or, Rabatel a beau jeu de montrer : (i) qu'on ne voit pas très bien la différence entre un « récit en focalisation interne variable » et un "récit en focalisation zéro reposant sur des focalisations internes variables »... (ii) qu'on ne saurait concevoir un récit «non focalisé », puisque toute description d'un référent fictionnel quelconque donne lieu par définition à la projection d'un point de vue (quel qu'il soit) sur ce référent, y compris dans les textes narrés à la troisième personne par un narrateur prétendument «impersonnel » censé adopter «le fameux "point de vue de Dieu" ou de Sirius » (Genette 1983 : 49).

(b) Où se situe le foyer perceptionnel dans une focalisation externe? Rabatel nie l'existence même de la focalisation externe, en démontrant: (i) que ce concept découle d'une confusion entre positionnement $\mathrm{du}$ focalisateur (le focalisateur est censé être se situer «hors de tout personnage ", Genette 1983 : 50) et modalités de description du focalisé (le référent qui fait l'objet d'une focalisation externe est vu de l'extérieur); (ii) qu'il n'a aucun fondement linguistique, puisque, dans les récits littéraires, il ne peut y avoir de «foyer » de la perspective distinct à la fois des personnages et du narrateur, seules instances énonciatrices susceptibles de constituer un centre de perspective. En toute logique, donc, Rabatel conclut que le point de vue narratif censé correspondre à une focalisation externe n'est en réalité qu'une vision externe d'un focalisé donné. 
AUTHOR

ILIAS YOCARIS

Université Côte d'Azur - LIRCES (EA 3159) 